Proyecciones Journal of Mathematics

Vol. 36, No 4, pp. 739-751, December 2017.

Universidad Católica del Norte

Antofagasta - Chile

\title{
Minimal open sets on generalized topological
}

\section{space}

\author{
Carlos Carpintero \\ Universidad de Oriente, Venezuela \\ Ennis Rosas \\ Universidad de la Costa, Colombia \\ Margot Salas-Brown \\ Universidad de Oriente, Venezuela \\ and \\ José Sanabria \\ Universidad de Oriente, Venezuela \\ Received: April 2017. Accepted : September 2017
}

\begin{abstract}
We introduce the notion of minimal open sets in a generalized topological space $(X, \mu)$. We investigate some of their fundamental properties and proved that any subset of a minimal open set on a $G T S(X, \mu)$ is a $\mu$-preopen set.
\end{abstract}

Keywords: Generalized topology, minimal $\mu$-open sets, $\mu$-pre-Hausdorff space.

Subjclass [2010]: $54 C 10,54 D 10$. 


\section{Introduction}

Generalized topological spaces and generalized open sets play a very important role in almost all branches of pure and applied mathematics, specially in General Topology, indeed for the last one decade or so, the research are concerned with the investigations of generalized topological spaces and several classes of generalized types of open sets.

The concepts of minimal open sets and maximal closed sets in topological spaces were introduced and considered by Nakaoka and Oda in [6] and [7]. More precisely, in 2001, Nakaoka and Oda [7] characterized the notions of minimal open sets and proved that any subset of a minimal open set is preopen. Also, as an application of a theory of minimal open sets, they obtained a sufficient condition for a locally finite space to be a pre-Hausdorff space. The purpose of this paper is to introduce a new class of $\mu$-open sets called minimal $\mu$-open set in generalized topologies and investigate some of their fundamental properties and proved that any subset of a minimal open set on a $\operatorname{GTS}(X, \mu)$ is a $\mu$-preopen set. Finally, we proof that this new concept of minimal $\mu$-open set in generalized topologies, generalize the concepts of minimal open [7] (respectively minimal $\gamma$-open [2], minimal $\gamma$ semiopen [3], minimal $I$-open [11], minimal $\lambda_{c^{-}}$open [5], minimal $P_{\gamma}$-open [4]) set.

Let $X$ be a nonempty set and $2^{X}$ denotes the power set of $X$. Then $\mu \subset 2^{X}$ is called a generalized topology (briefly GT) on $X$ [1] if and only if $\emptyset \in \mu$ and $G_{i} \in \mu$ for $i \in I \neq \emptyset$ implies $\bigcup_{i \in I} G_{i} \in \mu$. We call the pair $(X, \mu)$ a generalized topological space (briefly GTS). The elements of $\mu$ are called $\mu$-open sets and the complements are called $\mu$-closed sets. The generalized closure of a subset $A$ of $X$, denoted by $c_{\mu}(A)$, is the intersection of all $\mu$-closed sets containing $A$, and the generalized interior of $A$, denoted by $i_{\mu}(A)$, is the union of all $\mu$-open sets contained in $A$. A subset $M$ of a $\operatorname{GTS}(X, \mu)$ is called a $\mu$-preopen set if $M \subset i_{\mu}\left(c_{\mu}(M)\right)$. It is observed that a large number of papers are devoted to the study of $\mu$-open sets like open sets of topological space, possessing properties more or less similar to those of open sets as we can mention some of them:

1. if $A \subseteq B$, then $c_{\mu}(A) \subseteq c_{\mu}(B)$ and $i_{\mu}(A) \subseteq i_{\mu}(B)$.

2. if $U \in \mu$, then $i_{\mu}(U)=U$.

3. for any $A \subset X, c_{\mu}\left(c_{\mu}(A)\right)=c_{\mu}(A), A \subseteq c_{\mu}(A)$ and $i_{\mu}(A) \subseteq A$.

4. for any $A, B$ subsets of $X, c_{\mu}(A) \cup c_{\mu}(B) \subseteq c_{\mu}(A \cup B)$. 


\section{Minimal $\mu$-open sets}

Definition 2.1. A proper nonempty $\mu$-open set $U$ of a $\operatorname{GTS}(X, \mu)$ is called a minimal $\mu$-open set if $U \cap W=\emptyset$ or $U \subset W$ for any $W \in \mu$.

Remark 2.2. Observe that in a GTS $(X, \mu)$. If a proper nonempty subset $U$ of $X$ is not a minimal $\mu$-open set, then there exists a nonempty $\mu$-open set $V$ such that $V \cap U \neq \emptyset$ and $V \nsubseteq U$.

The following example shows a generalized topological space with a minimal $\mu$-open set.

Example 2.3. Let $\mathbf{R}$ be the set of real numbers and the GT

$$
\mu=\{\emptyset, \mathbf{R},\{\mathbf{0}\}\} \cup\{\mathbf{R} \backslash\{\mathbf{x}\}: \mathbf{x} \neq \mathbf{0}\} .
$$

that $\{0\}$ is a minimal $\mu$-open.

In topological spaces, Nakaoka and Oda [7], defined the minimal open sets as follows: A nonempty open set $U$ of $X$ is said to be a minimal open set if and only if any open set which is contained in $U$ is $\emptyset$ or $U$. In our case, the definition of minimal open sets in generalized topologies is the characterization given by Nakaoka and Oda [7] in Lemma 2.2(i), the reason is that in generalized topologies, we don't need stability for the intersection and we can obtain similar results on generalized topologies of minimal $\mu$-open sets.

Remark 2.4. It is important to note that in the article [8], authors work with maximal $\mu$-open and minimal $\mu$-closed sets, however, in the article under development, we study minimal $\mu$-open sets. Now these notions are independent as we can see:

In Example 2.4 of [8], the set $A=\{a, b\}$ is maximal $\mu$-open but is not minimal $\mu$-open according with Definition 2.1; in the same form, the set $B=\{c\}$ is not maximal $\mu$-open but it is minimal $\mu$-open according with Definition 2.1. In consequence, both concepts are independent. In the same form, the concepts of minimal $\mu$-closed set [8] and minimal $\mu$-open according with Definition 2.1 are independent.

Theorem 2.5. Let $U$ be a minimal $\mu$-open set of a $\operatorname{GTS}(X, \mu)$, then any $\mu$-open set contained in $U$ is $\emptyset$ or $U$. 
The following example shows, the converse of Theorem 2.5 is not necessarily true.

Example 2.6. Let $\mathbf{R}$ be the set of real numbers and

$$
\mu=\{\emptyset\} \cup\{A \subset \mathbf{R}:(\mathbf{0}, \mathbf{1}] \subset \mathbf{A} \text { or }[\mathbf{1}, \mathbf{2}) \subset \mathbf{A}\} .
$$

Then, $\mu$ is a $G T$ on $\mathbf{R}$. Observe that $(0,1] \in \mu$ and the only $\mu$-open sets contained in $(0,1]$ are $\emptyset$ and $(0,1]$ itself, while $(0,1]$ is not a minimal $\mu$-open, because $[1,2)$ is a $\mu$-open set such that $(0,1] \cap[1,2) \neq \emptyset$ and $(0,1]$ is not contained in $[1,2)$.

Now, if we consider a topological space $(X, \tau)$ and choose an adequate generalized topology $\mu$ on $X$, we obtain well known classes of minimal open sets using the Definition 2.1 as we can see.

1. The notion of minimal open set [7], is obtained when we choose the GT $\mu$ as a topology on $X$.

2. The notion of minimal $\gamma$-open [2], is obtained when we choose the GT $\mu$ as the collection of all $\gamma$-open subsets of $X$.

3. The notion of minimal $\gamma$-semiopen [3], is obtained when we choose the GT $\mu$ as the collection of all $\gamma$-semiopen subsets of $X$.

4. The notion of minimal $I$-open [11], is obtained when we choose the GT $\mu$ as the collection of all $I$-open subsets of $X$.

5. The notion of minimal $\lambda_{c}$-open [5], is obtained when we choose the GT $\mu$ as the collection of all $\lambda_{c^{-}}$open subsets of $X$.

6. The notion of minimal $P_{\gamma}$-open [4], is obtained when we choose the GT $\mu$ as the collection of all $P_{\gamma}$-open subsets of $X$.

Remark 2.7. Note that our definition of minimal $\mu$-open set in a generalized topology, generalize a well known classes of minimal open sets that appear in the literatura, as we can see: [7], [2], [3], [11], [5], [4]. Also with this new definition of minimal $\mu$-open set in a generalized topology, we can introduce new classes of minimal open sets, for example the class of minimal semi open sets [9] or the class of minimal pre-open sets [10]. In the first case, choose the GT $\mu$ as the collection of all semiopen subsets of $X$. In the second case, choose the GT $\mu$ as the collection of all preopen subsets of $X$.

Theorem 2.8. Let $U$ be a minimal $\mu$-open set of a $\operatorname{GTS}(X, \mu)$. If $x$ is an element of $U$, then $U \subset W$ for any $\mu$-open $W$ such that $x \in W$. 
Proof. If $W$ is a $\mu$-open set such that $x \in W$, then $U \cap W \neq \emptyset$. Thus, by Definition 2.1, $U \subset W$.

Theorem 2.9. Let $U$ be a minimal $\mu$-open set of a $\operatorname{GTS}(X, \mu)$. Then,

$$
U=\bigcap\{W: W \in \mu \text { and } x \in W\}
$$

for any element $x$ of $U$.

Proof. By Theorem 2.8, $U \subset W$ for any $\mu$-open $W$ such that $x \in W$, hence $U \subset \bigcap\{W: W \in \mu$ and $x \in W\}$. Since $U$ is a $\mu$-open set containing $x$, the result follows.

Theorem 2.10. Let $U$ be a $\mu$-open set of a $\operatorname{GTS}(X, \mu)$. The following are equivalent:

(1) $U$ is a minimal $\mu$-open set.

(2) $U \subset c_{\mu}(S)$ for any nonempty subset $S$ of $U$.

(3) $c_{\mu}(U)=c_{\mu}(S)$ for any nonempty subset $S$ of $U$.

Proof. $\quad(1) \Rightarrow(2)$. Let $x \in U, S$ any nonempty subset of $U$ and $W$ any $\mu$-open set containing $x$, then $U \subset W$ and hence $S=U \cap S \subset W \cap S$. Then, $W \cap S \neq \emptyset$ and therefore $x$ is an element of $c_{\mu}(S)$. It follows that $U \subset c_{\mu}(S)$.

$(2) \Rightarrow(3)$. For any nonempty subset $S$ of $U, c_{\mu}(S) \subset c_{\mu}(U)$. On the other hand, by $(2), c_{\mu}(U) \subset c_{\mu}\left(c_{\mu}(S)\right)=c_{\mu}(S)$. Therefore, $c_{\mu}(U)=c_{\mu}(S)$ for any nonempty subset $S$ of $U$.

$(3) \Rightarrow(1)$. Suppose that $U$ is not a minimal $\mu$-open set. Then by Remark 2.2 , there exists a nonempty $\mu$-open set $V$ such that $V \cap U \neq \emptyset$ and $V \nsubseteq U$. Hence, there exists an element $a \in V$ such that $a \notin U$. Then we have $c_{\mu}(\{a\}) \subset X \backslash U$. By $(3), c_{\mu}(\{a\})=c_{\mu}(V)$, hence $U \cap V \subset U \cap c_{\mu}(V)=$ $U \cap c_{\mu}(\{a\}) \subset U \cap X \backslash U=\emptyset$, and therefore $V \cap U=\emptyset$, which is a contradiction.

Theorem 2.11. Let $U$ be a minimal $\mu$-open set of a $\operatorname{GTS}(X, \mu)$. Then any nonempty subset $S$ of $U$ is a $\mu$-preopen set. 
Proof. $\quad$ By Theorem 2.10(2), $U \subset c_{\mu}(S)$ for any nonempty subset $S$ of $U$. It follows that $S \subset i_{\mu}\left(c_{\mu}(S)\right)$, because then $U=i_{\mu}(U) \subset i_{\mu}\left(c_{\mu}(S)\right)$.

Example 2.12. Let $\mathbf{R}$ be the set of real numbers and the GT

$$
\mu=\{\emptyset, \mathbf{R}, \mathbf{Q}\} \cup\{\mathbf{R} \backslash\{\mathbf{x}\}: \mathbf{x} \in(\mathbf{R} \backslash \mathbf{Q}) \cup\{\mathbf{0}\}\} .
$$

Observe that all subsets of $\mathbf{Q}$ are $\mu$-preopen but $\mathbf{Q}$ is not a minimal $\mu$-open set.

Theorem 2.13. Let $(X, \mu)$ be a GTS, $U$ be a nonempty $\mu$-open set and $M$ any nonempty subset of $X$. If there exists a $\mu$-open $W$, such that $M \subset W$ and $W \subset c_{\mu}(M) \cup c_{\mu}(U)$, then $M \cup S$ is a $\mu$-preopen set for any nonempty subset $S$ of $U$.

Proof. $\quad$ By Theorem $2.10(3), c_{\mu}(S)=c_{\mu}(U)$. Since

$$
M \subset W \subset c_{\mu}(M) \cup c_{\mu}(U)=c_{\mu}(M) \cup c_{\mu}(S) \subset c_{\mu}(M \cup S),
$$

therefore, $M \subset W=i_{\mu}(W) \subset i_{\mu}\left(c_{\mu}(M \cup S)\right)$.

On the other hand, $S \subset U \subset c_{\mu}(U) \subset c_{\mu}(M) \cup c_{\mu}(U) \subset c_{\mu}(M \cup S)$, and $S \subset i_{\mu}(U) \subset i_{\mu} c_{\mu}(M \cup S)$. Therefore, $M \cup S \subset i_{\mu}\left(c_{\mu}(M \cup S)\right)$.

Corollary 2.14. Let $(X, \mu)$ be a GTS, $U$ be a nonempty $\mu$-open set and $M$ any nonempty subset of $X$. If there exists a $\mu$-open $W$, such that $M \subset W$ and $W \subset c_{\mu}(U)$, then $M \cup S$ is a $\mu$-preopen set for any nonempty subset $S$ of $U$.

Proof. By hypothesis, $W \subset c_{\mu}(U) \subset c_{\mu}(M) \cup c_{\mu}(U)$. So by Theorem $2.13, M \cup S$ is a $\mu$-preopen set.

The following corollary is an immediate consequence of Definition 2.1.

Corollary 2.15. Let $U$ be a minimal $\mu$-open set of a $\operatorname{GTS}(X, \mu)$. If $x \in X$ and $U_{x}=\bigcap\{W: W \in \mu, x \in W\}$, then $U_{x} \cap U=\emptyset$ or $U \subset U_{x}$.

Proof. Let $U_{x}=\bigcap\{W: W \in \mu, x \in W\}$, then $U \cap U_{x}=\bigcap\{U \cap W$ : $W \in \mu, x \in W\}$. Observe that, by Definition 2.1, $U \cap W=\emptyset$ or $U \subset W$ for any $\mu$-open set $W$, then $U_{x} \cap U=\emptyset$ or $U \subset U_{x}$. 


\section{Finite $\mu$-open sets}

In this section, we study some properties of minimal $\mu$-open sets in finite $\mu$-open set and $\mu$-locally finite spaces.

Definition 3.1. Let $(X, \mu)$ be a GTS, we say that $X$ satisfies the generalized interior nonempty property (briefly g.i.n.e. property) if for any finite collection $\left\{U_{i}\right\}_{i=1}^{n}$ of nonempty $\mu$-open sets such that $\bigcap_{i=1}^{n} U_{i} \neq \emptyset$, satisfies $i_{\mu}\left(\bigcap_{i=1}^{n} U_{i}\right) \neq \emptyset$.

Example 3.2. Every topology is a GT that satisfies the g.i.n.e. property.

Now, we have in the following example a GT that satisfies the g.i.n.e. property but it is not a topology.

Example 3.3. Let $\mathbf{R}$ be the set of real numbers and the GT

$$
\mu=\{\emptyset, \mathbf{R},\{\mathbf{0}\}\} \cup\{\mathbf{R} \backslash\{\mathbf{x}\}: \mathbf{x} \neq \mathbf{0}\} .
$$

Observe that if $\left\{A_{i}\right\}_{i=1}^{n}$ is a collection of nonempty elements of $\mu$ then $0 \in \bigcap_{i=1}^{n} A_{i}$ and therefore $i_{\mu}\left(\bigcap_{i=1}^{n} A_{i}\right) \neq \emptyset$, but $\mu$ is not a topology, because, if we take $\mathbf{R} \backslash\{\mathbf{1}\}$ and $\mathbf{R} \backslash\{\mathbf{2}\},(\mathbf{R} \backslash\{\mathbf{1}\}) \cap(\mathbf{R} \backslash\{\mathbf{2}\})=\mathbf{R} \backslash\{\mathbf{1}, \mathbf{2}\}$ is not a $\mu$-open set.

Theorem 3.4. Let $(X, \mu)$ be a GTS that satisfies the g.i.n.e. property and $V$ be a nonempty finite set of $X$ such that $i_{\mu}(V) \neq \emptyset$. Then there exists at least one (finite) minimal $\mu$-open set $U$ such $U \subset i_{\mu}(V)$.

Proof. If $i_{\mu}(V)$ is a minimal $\mu$-open set, write $U=i_{\mu}(V)$. If $i_{\mu}(V)$ is not a minimal $\mu$-open set, then there exists a $\mu$-open set $W$ such that $i_{\mu}(V) \cap W \neq \emptyset$ and $i_{\mu}(V) \nsubseteq W$. Observe $i_{\mu}(V) \cap W$ is a proper subset of $i_{\mu}(V)$ and $i_{\mu}(V), W$ are nonempty $\mu$-open sets with $i_{\mu}(V) \cap W \neq \emptyset$. Take $V_{1}=i_{\mu}(V \cap W)$, then $V_{1}$ is a finite $\mu$-open set and $V_{1}$ is a proper subset of $i_{\mu}(V)$. Now using the g.i.n.e. property, we obtain that $V_{1} \neq \emptyset$. If $V_{1}$ is minimal $\mu$-open, write set $U=V_{1}$.

If $V_{1}$ is not a minimal $\mu$-open, continuing with the process, there exists a finite $\mu$-open set $V_{2}$ such that $\emptyset \neq V_{2}$ is a proper subset of $V_{1}$ and $V_{1}$ is a proper subset of $V$. Continuing this process, we have a sequence of $\mu$-open sets

$$
i_{\mu}(V) \supset V_{1} \supset V_{2} \supset \ldots \supset V_{k} \supset \ldots
$$


Since $i_{\mu}(V)$ is a finite set, this process only we can do it finitely. Then, finally we get a minimal $\mu$-open set $U=V_{n}$ for some positive integer n.

The following example shows that the g.i.n.e. property can not be removed in the Theorem 3.4.

Example 3.5. Let $\mathbf{R}$ be the set of real numbers and the GT,

$$
\mu=\{\emptyset\} \cup\{A \subset \mathbf{R}:|\mathbf{A}| \geq \mathbf{3}\},
$$

where $|A|$ denote the cardinality of $A$. Observe that $(\mathbf{R}, \mu)$ does not satisfy the g.i.n.e. property. If we take $A=\{1,2,3,4\}$ then $i_{\mu}(A)=A \neq \emptyset$ but $A$ does not contain any minimal $\mu$-open set.

Definition 3.6. A GTS $(X, \mu)$ is said to be $\mu$-locally finite if any of its points is contained in a finite $\mu$-open set.

Example 3.7. Consider $\mathbf{R}$ with the GT

$$
\mu=\{A \subset \mathbf{R}: \mathbf{0} \in \mathbf{A} \text { or } \mathbf{1} \in \mathbf{A}\} \cup\{\emptyset\} .
$$

Observe that $(\mathbf{R}, \mu)$ is $\mu$-locally finite, because for each $x \in \mathbf{R},\{x, 0\}$ is a finite $\mu$-open set containing $x$.

Theorem 3.8. Let $(X, \mu)$ be a GTS that is $\mu$-locally finite and satisfies the g.i.n.e. property. Let $V$ be any nonempty $\mu$-open set. Then there exists at least one finite minimal $\mu$-open set $U$ such that $U \subset V$.

Proof. Since $V$ is a nonempty set, there exists an element $x$ of $V$. Since $X$ is $\mu$-locally finite and $(X, \mu)$ satisfies the g.i.n.e. property, we have a finite $\mu$-open set $V_{x}$ such that $x \in V_{x}$. Since $V \cap V_{x}$ is a finite set and nonempty, then using Theorem 3.4, we get a minimal $\mu$-open set $U$ such that $U \subset i_{\mu}\left(V \cap V_{x}\right) \subset V \cap V_{x} \subset V$.

Theorem 3.9. Let $(X, \mu)$ be a GTS that satisfies the g.i.n.e. property and $U$ any nonempty finite $\mu$-open set which is not a minimal $\mu$-open set. If $\left\{U_{1}, U_{2}, \ldots, U_{n}\right\}$ is the set of all minimal $\mu$-open set in $U$ and $x$ is an element of $U \backslash\left(U_{1} \cup U_{2} \cup \ldots \cup U_{k}\right)$ and $U_{x}=\bigcap\{W: W \in \mu, x \in W\}$, then there exists a positive integer $i \in\{1, \ldots, n\}$ such that $U_{i} \subset U_{x}$. 
Proof. Assume that $U_{i} \nsubseteq \nsubseteq U_{x}$ for any positive integer $i \in\{1, \ldots, n\}$. By Corollary 2.15, $U_{i} \cap U_{x}=\emptyset$ for any minimal $\mu$-open $U_{i} \subset U$. Since $U_{x} \subset U$, hence $U_{x}$ is a finite nonempty set and $i_{\mu}\left(U_{x}\right) \neq \emptyset$. By Theorem 3.4, there exists a nonempty minimal $\mu$-open set $U^{\prime}$ such that $U^{\prime} \subset U_{x}$. Since $U^{\prime} \subset$ $U_{x} \subset U, U^{\prime}$ is a minimal $\mu$-open set in $U$. Follows that $U_{i} \cap U^{\prime} \subset U_{i} \cap U_{x}=\emptyset$ for any minimal $\mu$-open set $U_{i}$. Therefore $U^{\prime} \neq U_{i}$ for any positive integer $i$ of $\{1, \ldots, n\}$. This contradicts our assumption.

Corollary 3.10. Let $(X, \mu)$ be a GTS that satisfies the g.i.n.e. property and $U$ a finite nonempty $\mu$-open set which is not a minimal $\mu$-open set. If $\left\{U_{1}, U_{2}, \ldots, U_{n}\right\}$ is the set of all minimal $\mu$-open set in $U$ and $x$ an element of $U \backslash\left(U_{1} \cup U_{2} \cup \ldots \cup U_{n}\right)$, then there exists a positive integer $i \in\{1, \ldots, n\}$ such that $U_{i} \subset W_{x}$ for any $\mu$-open $W_{x}$ containing $x$.

Proof. Observe that $W_{x}=\bigcap\{W: W \in \mu, x \in W\}$, then the result follows from Theorem 3.9.

Corollary 3.11. Let $(X, \mu)$ be a GTS that satisfies the g.i.n.e. property and $U$ be a nonempty finite $\mu$-open set which is not a minimal $\mu$-open set. If $\left\{U_{1}, U_{2}, \ldots, U_{n}\right\}$ is the set of all minimal $\mu$-open set in $U$ and $x$ an element of $U \backslash\left(U_{1} \cup U_{2} \cup \ldots \cup U_{n}\right)$, then there exists a positive integer $i \in\{1, \ldots, n\}$ such that $x$ is an element of $c_{\mu}\left(U_{i}\right)$.

Proof. By Corollary 3.10, there exists a positive integer $i \in\{1, \ldots, n\}$ such that $U_{i} \subset W$ for any $\mu$-open set $W$ containing $x$. Therefore $U_{i} \cap W \neq \emptyset$ for any $\mu$-open set $W$ such that $x \in W$. And the result follows.

Theorem 3.12. Let $(X, \mu)$ be a GTS that satisfies the g.i.n.e. property, $U$ be a nonempty finite $\mu$-open set and for each $i \in\{1, \ldots, n\}, U_{i}$ is a minimal $\mu$-open set contained in $U$. The following are equivalent:

(1) $\left\{U_{1}, U_{2}, \ldots, U_{n}\right\}$ is the set of all minimal $\mu$-open sets in $U$.

(2) $U \subset c_{\mu}\left(S_{1} \cup S_{2} \cup \ldots \cup S_{n}\right)$ for any nonempty subset $S_{i}$ of $U_{i}$ for $i \in\{1, \ldots, n\}$.

(3) $c_{\mu}(U)=c_{\mu}\left(S_{1} \cup S_{2} \cup \ldots \cup S_{n}\right)$ for any nonempty subset $S_{i}$ of $U_{i}$ for $i \in\{1, \ldots, n\}$. 
Proof. $\quad(1) \Rightarrow(2)$. If $U$ is a minimal $\mu$-open set, by Theorem 2.10, $U \subset$ $c_{\mu}\left(S_{i}\right)$ for any subset $S_{i}$ of $U_{i}$ with $i \in\{1, \ldots, n\}$. Therefore

$$
U \subset c_{\mu}\left(S_{1}\right) \cup c_{\mu}\left(S_{2}\right) \cup \ldots \cup c_{\mu}\left(S_{n}\right) \subset c_{\mu}\left(S_{1} \cup S_{2} \cup \ldots \cup S_{n}\right) .
$$

Otherwise, if $U$ is not a minimal $\mu$-open set, and $x$ is any element of $U-\left(U_{1} \cup U_{2} \cup \ldots \cup U_{n}\right)$, by Theorem 3.11, there exists a positive integer $i \in\{1, \ldots, n\}$ such that $x \in c_{\mu}\left(U_{i}\right) \subset c_{\mu}\left(U_{1}\right) \cup c_{\mu}\left(U_{2}\right) \cup \ldots \cup c_{\mu}\left(U_{n}\right)$. Therefore, by Theorem 2.10,

$$
\begin{aligned}
U \subset c_{\mu}\left(U_{1}\right) \cup c_{\mu}\left(U_{2}\right) \cup \ldots \cup c_{\mu}\left(U_{n}\right)=c_{\mu}\left(S_{1}\right) \cup c_{\mu}\left(S_{2}\right) \cup \ldots \cup c_{\mu}\left(S_{n}\right) \subset \\
c_{\mu}\left(S_{1} \cup S_{2} \cup \ldots \cup S_{n}\right) .
\end{aligned}
$$

$(2) \Rightarrow(3)$. For any nonempty subset $S_{i}$ of $U_{i}$ with $i \in\{1, \ldots, n\}$, we have

$$
S_{1} \cup S_{2} \cup \ldots \cup S_{n} \subset U_{1} \cup U_{2} \cup \ldots \cup U_{n} \subset U
$$

and therefore, $c_{\mu}\left(S_{1} \cup S_{2} \cup \ldots \cup S_{n}\right) \subset c_{\mu}(U)$. On the other hand, by (2), we see

$$
c_{\mu}(U) \subset c_{\mu}\left(c_{\mu}\left(S_{1} \cup S_{2} \cup \ldots \cup S_{n}\right)\right)=c_{\mu}\left(S_{1} \cup S_{2} \cup \ldots \cup S_{n}\right) .
$$

Therefore, $c_{\mu}(U)=c_{\mu}\left(S_{1} \cup S_{2} \cup \ldots \cup S_{n}\right)$ for any nonempty subset $S_{i}$ of $U_{i}$ with $i \in\{1, \ldots, n\}$.

$(3) \Rightarrow(1)$. Suppose that $V$ is a minimal $\mu$-open set in $U$ and $V \neq U_{i}$ with $i \in\{1, \ldots, n\}$. Then $V \cap c_{\mu}\left(U_{i}\right)=\emptyset$ for each $i \in\{1, \ldots, n\}$. It follows that, any element of $V$ is not contained in $c_{\mu}\left(U_{1} \cup U_{2} \cup \ldots \cup U_{n}\right)$. This contradicts the condition (3), because $V \subset U \subset c_{\mu}(U)=c_{\mu}\left(S_{1} \cup S_{2} \cup \ldots \cup S_{n}\right)$.

Theorem 3.13. Let $(X, \mu)$ be a GTS that satisfies the g.i.n.e. property, $U$ a nonempty finite $\mu$-open set and $\left\{U_{1}, U_{2}, \ldots, U_{n}\right\}$ the collection of all minimal $\mu$-open sets in $U$. If $S$ is any subset of $U \backslash\left(U_{1} \cup U_{2} \cup \ldots \cup U_{n}\right)$ and $S_{i}$ is any nonempty subset of $U_{i}$ for each $i \in\{1, \ldots, n\}$, then $S \cup S_{1} \cup S_{2} \cup \ldots \cup S_{n}$ is a $\mu$-preopen set.

Proof. By Theorem 3.12(2),

$$
U \subset c_{\mu}\left(S_{1} \cup S_{2} \cup \ldots \cup S_{n}\right) \subset c_{\mu}\left(S \cup S_{1} \cup S_{2} \cup \ldots \cup S_{n}\right) .
$$

Since $U$ is a $\mu$-open set, then

$$
S \cup S_{1} \cup S_{2} \cup \ldots \cup S_{n} \subset U=i_{\mu}(U) \subset i_{\mu}\left(c_{\mu}\left(S \cup S_{1} \cup S_{2} \cup \ldots \cup S_{n}\right)\right) .
$$


And the result follows.

Definition 3.14. Let $(X, \mu)$ be a GTS. $X$ is said to be $\mu$-pre-Hausdorff space if for each pairs $x, y \in X$, with $x \neq y$, there exist $\mu$-preopen sets $U, V$ such that $x \in U, y \in V$ and $U \cap V=\emptyset$.

Example 3.15. The GTS given in the Examples 3.5 and 3.7 are $\mu$-preHausdorff spaces.

Example 3.16. The GTS given in Example 2.3 is not a $\mu$-pre-Hausdorff space.

Theorem 3.17. Let $(X, \mu)$ be a GTS $\mu$-locally finite that satisfies the g.i.n.e. property. If any minimal $\mu$-open set of $X$ has at least two elements, then $X$ is a $\mu$-pre-Hausdorff space.

Proof. Let $x, y$ be two elements of $X$ such that $x \neq y$. Since $X$ is $\mu$-locally finite space, there exist a finite $\mu$-open sets $U$ and $V$ such that $x \in U$ and $y \in V$. By Theorem 3.4, consider $\left\{U_{1}, U_{2}, \ldots, U_{n}\right\}$ the set of all minimal $\mu$-open sets in $U$ and $\left\{V_{1}, V_{2}, \ldots, V_{m}\right\}$ the set of all minimal $\mu$-open set in $V$. Then we analyze the following three cases:

Case 1. If there exists $i \in\{1, \ldots, n\}$ and $j \in\{1,2, \ldots, m\}$ such that $x \in U_{i}$ and $y \in V_{j}$, then, by Theorem 2.11, $\{x\}$ and $\{y\}$ are disjoint $\mu$-preopen sets containing $x$ and $y$, respectively, the result follows.

Case 2. If there exists $i \in\{1, \ldots, n\}$ such that $x \in U_{i}$ and $y \notin V_{i}$ for any $j \in\{1, \ldots, m\}$, then we find an element $y_{j}$ of $V_{j}$ for each $j \in$ $\{1, \ldots, m\}$ such that $\{x\}$ and $\left\{y, y_{1}, y_{2}, \ldots, y_{m}\right\}$ are $\mu$-preopen sets and $\{x\} \cap$ $\left\{y, y_{1}, y_{2}, \ldots, y_{m}\right\}=\emptyset$. By Theorems 2.11 and 3.13 and the hypothesis, the result follows.

Case 3. If $x \notin U_{i}$ for any $i \in\{1, \ldots, n\}$ and $y \notin V_{i}$ for any $j \in$ $\{1, \ldots, m\}$, then we find elements $x_{i} \in U_{i}$ and $y_{j} \in V_{i}$ for each $i, j$ such that $\left\{x, x_{1}, x_{2}, \ldots, x_{n}\right\}$ and $\left\{y, y_{1}, y_{2}, \ldots, y_{m}\right\}$ are $\mu$-preopen sets and $\left\{x, x_{1}, x_{2}, \ldots, x_{n}\right\} \cap$ $\left\{y, y_{1}, y_{2}, \ldots, y_{m}\right\}=\emptyset$. By Theorem 3.13 and the hypothesis, the result follows.

\section{References}

[1] . Császár, Generalized topology, generalized continuity. Acta Math. Hungar., 96, pp. 351-357, (2002). 
[2] S. Hussain and B. Ahmad, On minimal $\gamma$-open sets. EJPAM., 2 (3), pp. 338-351, (2009).

[3] S. Hussain and B. Ahmad, $\gamma$-semi-open sets in topological spaces II. Southeast Asian Bull. Math., 34 (5), pp. 997-1008, (2010).

[4] A. B. Khalaf and H. Z. Ibrahim, Some applications of minimal $P_{\gamma}$-open sets. Demonstr. Math., 47 (2), pp. 423-432, (2014).

[5] A. B. Khalaf and S. F. Namiq, On minimal $\lambda_{c}$-open sets. Asian. J. Fuzzy. Applied Math., 2, pp. 198-202, (2014).

[6] F. Nakaoka and N. Oda, Minimal closed sets and maximal closed sets. Int. J. Math. Math. Sci., pp. 1-8, (2006).

[7] F. Nakaoka and N. Oda, Some applications of minimal open sets. Int. J. Math. Math. Sci., 27 (8), pp. 471-476, (2001).

[8] B. Roy and R. Sen, On maximal $\mu$-open and minimal $\mu$-closed sets via generalized topology. Acta Math. Hungar., 136 (4), pp. 233-239, (2012).

[9] M. Salas B., E. Rosas, C. Carpintero and J. E. Sanabria, On minimal semi open sets on generalized topology. Under preparation.

[10] M. Salas B., E. Rosas, C. Carpintero and J. E. Sanabria, On minimal pre open sets on generalized topology. Under preparation.

[11] A. Vadivel, R. Aishwarya and M. Saraswathi, Minimal $\mathcal{I}$-open sets and maximal $\mathcal{I}$-closed sets, J. of Adv. Research in Pure Math., Vol. 3, 1, pp. 87-95, (2011).

\section{Carlos Carpintero}

Departamento de Matemáticas

Universidad De Oriente

Cumaná

Venezuela

e-mail : carpintero.carlos@gmail.com 


\section{Ennis Rosas}

Departamento de Ciencias Naturales y Exactas

Universidad de la Costa

Barranquilla

Colombia

e-mail : ennisrafael@gmail.com

\section{Margot Salas-Brown}

Departamento de Matemáticas

Universidad De Oriente

Cumaná

Venezuela

e-mail : salasbrown@gmail.com

and

\section{José Sanabria}

Departamento de Matemáticas

Universidad De Oriente

Cumaná

Venezuela

e-mail : jesanabri@gmail.com 